\title{
Reactive arthritis-associated aortitis followed by Yersinia Enterocolitica infection: Multimodal imaging
}

\author{
Rafał Gałąska ${ }^{1}$, Anna Masiak², Dorota Kulawiak-Gałąska ${ }^{3}$, Zenobia Czuszyńska², \\ Karolina Dorniak ${ }^{4}$, Marcin Fijałkowski ${ }^{1}$ \\ ${ }^{1} 1^{\text {st }}$ Department of Cardiology, Medical University of Gdansk, Poland \\ ${ }^{2}$ Chair and Department of Internal Diseases, Connective Tissue Diseases and Geriatrics, \\ Medical University of Gdansk, Poland \\ ${ }^{3}$ Department of Radiology, Medical University of Gdansk, Poland \\ ${ }^{4}$ Department of Noninvasive Cardiac Diagnostics, Medical University of Gdansk, Poland
}

A 51-year-old female was admitted to the Department of Cardiology due to severe aortic insufficiency. Her medical history revealed she had been treated 5 years earlier with naproxen and oral corticosteroids for seronegative arthritis. Echocardiography performed at that time revealed she had mild aortic insufficiency. Four years later when her arthritis recurred, after serological tests (IgA 85.2 U/mL, IgG 88.4 U/mL IgM 36.7 U/mL, positive over $25 \mathrm{U} / \mathrm{mL}$ ) she was diagnosed with yersiniosis. The patient was treated with ciprofloxacine ( 5 weeks) and ceftriaxone (another 3 weeks). Despite this treatment she still had active arthritis in her right wrist, nodular erythema and heel enthesopathy. Her C-reactive protein was persistently elevated $(21 \mathrm{mg} / \mathrm{dL})$. Echocardiography revealed severe aortic regurgitation with moderately decreased left ventricular ejection fraction and thickening of the wall of the ascendens aorta (Fig. 1A, B). Aortic wall involvement was confirmed by mulitdetector computed tomography, magnetic resonance examinations, and positron emission tomography (Fig. 1C, D, Suppl. Video 1). She was then diagnosed with noninfectious aortitis. The patient was treated with corticosteroids and cyclophospha- mide which resulted in a complete resolution of her rheumatic symptoms. Control mulitdetector computed tomography and magnetic resonance examinations revealed only partial withdrawal of the primary changes in the aorta (Fig. 1E, F) with regions of delayed enhancement in the wall of her ascendens aorta, aortic arch and its branches (Fig. 1G, H). Due to symptomatic severe aortic insufficiency, the aortic valve was replaced with a St. Jude 23 prothesis which resulted in significant improvement of exercise tolerance as well as left ventricular function.

Reactive arthritis is an immune-mediated seronegative arthritis that belongs to a group of spondyloarthropathies and develops after a gastrointestinal or genitourinary system infection. This patient is unique for several reasons. She presented isolated arthritis without involvement of eyes and sacroiliac joints and her HLA B27 test was negative. The opinion presented herein is that the Yersinia infection led to reactive arthritis as well as to aortitis and severe aortic insufficiency. Reactive arthritis has been considered a self-limited disease, but in fact symptoms can persist for many years and can lead to serious target organ complications.

Conflict of interest: None declared

Address for correspondence: Dr. Rafał Gałąska, $1^{\text {st }}$ Department of Cardiology, Medical University of Gdansk, ul. Dębinki 7 , 80-952 Gdańsk, Poland, tel: +48 5834925 04, fax: +485834612 01, e-mail: rgal@gumed.edu.pl 


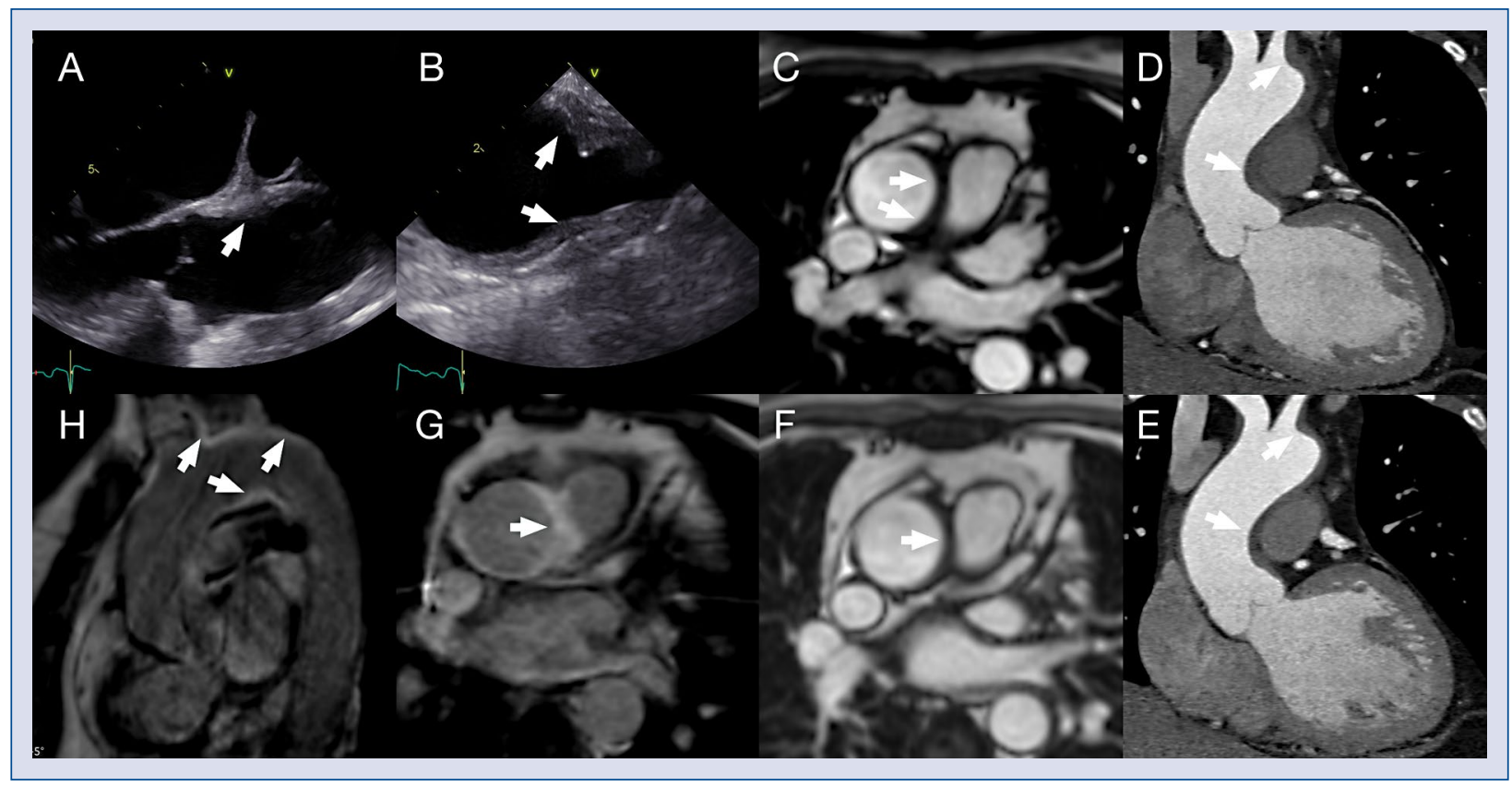

Figure 1. A. Transesophageal echocardiography, focal thickening of the ascendens aorta wall (arrows) before treatment; B. Transesophageal echocardiography, thickening of the wall of the aortic arch and proximal parts of its branches (arrows) before treatment; C. TrueFisp magnetic resonance examinations imaging of ascending aorta, note a semilunar thickening of the aortic wall before treatment (arrows); D. Mulitdetector computed tomography multiplanar reconstruction demonstrating a thickening of the wall of the ascending aorta and aortic arch before treatment; E. Mulitdetector computed tomography after treatment; F. TrueFisp magnetic resonance examination imaging after treatment; G. Delayed enhancement magnetic resonance examination imaging, note regions of the enhancement of the wall of the ascending aorta; $\mathbf{H}$. Delayed enhancement magnetic resonance examinations imaging, note regions of the enhancement of the wall of the aortic arch and its branches. 